\title{
Evolution of Optimal Control Policies for Manufacturing Systems
}

\author{
B. Porter \\ Department of Industrial and Manufacturing \\ Systems Engineering \\ The University of Hong Kong \\ Hong Kong
}

\begin{abstract}
Genetic algorithms provide a straightforward technique for the synthesis of optimal control policies for manufacturing systems. However, it is shown in this paper that punctuated equilibria can occur in the evolutionary processes associated with such genetic synthesis procedures. The care that must therefore be exercised in avoiding sub-optimal control policies is illustrated by reference to the genetic synthesis of optimal control policies for a simple manufacturing system.
\end{abstract}

\section{INTRODUCTION}

In contemporary industrial automation, the synthesis of optimal control policies for manufacturing systems is a crucially important task. However, the use of classical techniques such as dynamic programming [1] or the maximum principle [2] to synthesise such policies for such systems frequently leads to conceptual or computational difficulties. In order to remedy this situation, it was therefore shown by Porter and Allacui [3] that genetic algorithms [4] [5] provide a straightforward alternative technique for the synthesis of optimal control policies for manufacturing systems. In particular, it was shown [3] that this genetic procedure has the great merit that constraints on control and state vectors can be readily accommodated (simply by assigning zero Darwinian fitness to any control vector that violates these constraints).

However, it is shown in this paper that punctuated equilibria (similar to those that occur in natural evolutionary processes [6]) can occur in the simulated evolutionary processes associated with such genetic synthesis procedures. In such situations, the evolutionary process settles down in relatively long periods of stasis that are punctuated irregularly by brief periods of rapid evolutionary change. It follows that great care must be exercised in avoiding the suboptimal control policies corresponding to such periods of stasis which occur before the evolutionary process is complete. The need for this care is illustrated by

\author{
C. Allaoui \\ Research Institute for Design, Manufacture, and \\ Marketing \\ University of Salford \\ England
}

reference to the genetic synthesis of optimal control policies for a simple manufacturing syste $n$.

\section{GENETIC SYNTHESIS PROCEDURE}

The manufacturing systems under investigation comprise $m$ machines (with $n$ associated buffers) and produce $\mathrm{p}$ part types. The dynamical behaviour of such systems is governed by linear differential equation of the forms [7][8]

$$
\dot{q}(t)=A_{1} u(t)+A_{2} i(t)
$$

and

$$
\dot{x}(t)=A_{3} u(t)-d(t)
$$

for the buffer dynamics and for the production dynamics, respectively. In these equations, $q(t) \in \Re^{n}$ is the vector of buffer levels, $x(t) \in \mathfrak{R}^{p}$ is the vector of finished parts, $u(t) \in \Re^{n}$ is the vector of production rates, $i(t) \in \Re^{p}$ is the vector of part release rates, and $d(t) \in \Re^{p}$ is the vector of part demand rates. If $\tau_{j}$ is the processing time of parts in buffer $j$ and $B^{(k)}$ is the set of buffers for machine $k(k=1,2, . ., m)$, then the buffer production rates must satisfy the capacity constraints

$$
\sum_{j \in B^{(k)}} \tau_{j} u_{j}(t) \leq 1 \quad(k=1,2, \ldots, m)
$$

In addition, the state and control vectors must satisfy the constraints

$$
\begin{aligned}
& q(t) \geq 0, \\
& u(t) \geq 0,
\end{aligned}
$$




$$
\mathrm{i}(\mathrm{t}) \geq 0 \quad \text {. }
$$

However, $x(t)$, the state vector of finished parts, is not required to satisfy such a non-negativity constraint (since there may be either a surplus or a backlog of finished parts).

The control problem is to find, over some time period of duration $T$, the vectors $i(t) \in \Re^{p}$ and $u(t) \in \mathfrak{R}^{\mathfrak{n}}$ of part release rates and buffer production rates that minimise the cost function

$$
\Gamma=\int_{0}^{T}\left[\lambda q(t)+\mu^{+} x^{+}(t)+\mu^{-} x^{-}(t)\right] d t
$$

In equation (7),

$$
\mathrm{x}^{+}(\mathrm{t})=\max \{\mathrm{x}(\mathrm{t}), 0\} \in \mathfrak{R}^{\mathrm{p}}
$$

is the parts surplus vector,

$$
\mathrm{x}^{-}(\mathrm{t})=\max \{-\mathrm{x}(\mathrm{t}), 0\} \in \mathfrak{R}^{\mathrm{p}}
$$

is the parts backlog vector, and $\lambda \in \mathfrak{R}^{1 \mathrm{xn}}, \mu^{-} \in \mathfrak{R}^{1 \mathrm{xp}}, \mu^{+} \in \mathfrak{R}^{1 \mathrm{xp}}$ are weighting vectors for the buffer contents and the finished parts surplus or backlog. It is evident that the control vectors $i(t) \in \mathfrak{R}^{p}$ and $\mathrm{u}(\mathrm{t}) \in \mathfrak{R}^{\mathrm{n}}$ that minimise this cost function, $\Gamma$, are optimal in the sense that the entire cost function associated with work-in-progress (as measured by the buffer levels), production surplus, and production backlog is minimised. However, the solution of this optimisation problem is non-trivial because tine control vectors $\mathrm{i}(\mathrm{t}) \in \mathfrak{R}^{\mathrm{p}}$ and $\mathrm{u}(\mathrm{t}) \in \mathfrak{R}^{\mathrm{n}}$ must satisfy the constraints (3), (5), and (6) whilst the state vector $q(t) \in \Re^{n}$ must satisfy the constraint (4).

Nevertheless, the procedures for the genetic design of control systems described by Porter [4] can be readily used to solve this optimal control problem for manufacturing systems [3]. Thus, let the control interval $[0, T]$ be divided into $N$ sub-intervals, and let the elements of the vectors $i(t) \in \Re^{p}$ and $u(t) \in \Re^{n}$ be piecewise-constant functions on these sub-intervals. In this way, the entire control vector $\left[\mathrm{i}^{\mathrm{T}}(\mathrm{t}), \mathrm{u}^{\mathrm{T}}(\mathrm{t})\right]^{\mathrm{T}} \in \mathfrak{R}^{\mathrm{p}+\mathrm{n}}$ can be represented on the control interval $[0, T]$ by an ordered set of $(p+n) N$ numbers. Therefore, if each of these numbers is represented by a sub-string of binary digits, it is evident that the entire control vector $\left[i^{\mathrm{T}}(t), u^{\mathrm{T}}(t)\right]^{\mathrm{T}} \in \Re^{\mathrm{p}+\mathrm{n}}$ can be represented on the control interval $[0, T]$ by the string of binary digits formed by concatenating these $(p+n) N$ sub-strings. The Darwinian fitness of each such string is normally given by

$$
\Phi=\frac{1}{\Gamma}
$$

where $\Gamma$ is the value of the cost function (7) associated with the entire control vector. However, if any of the constraints (3), (4), (5), and (6) are violated, the Darwinian fitness is then given by

$$
\Phi=0
$$

These procedures for encoding entire control vectors as binary strings, and for determining the Darwinian fitness of each such string, greatly facilitate the use of genetic algorithms for determining the optimal entire control vector $\left[\mathrm{i}^{\mathrm{T}}(\mathrm{t}), \mathrm{u}^{\mathrm{T}}(\mathrm{t})\right]^{\mathrm{T}} \in \Re^{\mathrm{p}+\mathrm{n}}$. Thus, following an initial choice of population of such binary strings, successive generations of populations of strings can be rapidly obtained by using the standard genetic operations of selection, crossover, and mutation [5]. These genetic operations ensure that the successive generations of entire control vectors produced by the genetic algorithm exhibit progressively improving performance as the evolutionary process continues. In this way, the optimal entire control vector $\left[\mathrm{i}^{\mathrm{T}}(\mathrm{t}), \mathrm{u}^{\mathrm{T}}(\mathrm{t})\right]^{\mathrm{T}} \in \Re^{\mathrm{p}+\mathrm{n}}$ is eventually obtained which minimises the cost function, $\Gamma$, defined in equation (7) whilst respecting the constraints represented by the inequalities (3), (4), (5), and (6).

\section{ILLUSTRATTVE EXAMPLE}

The genetic synthesis of optimal control policies for manufacturing systems can be conveniently illustrated by considering a simple system in which $m=2, n=2$, and $p=1$. In this case, equation (1) for the buffer dynamics assumes the scalar forms 


$$
\dot{\mathrm{q}}_{1}(\mathrm{t})=\mathrm{i}(\mathrm{t})-\mathrm{u}_{1}(\mathrm{t})
$$

and

$$
\dot{\mathrm{q}}_{2}(\mathrm{t})=\mathrm{u}_{1}(\mathrm{t})-\mathrm{u}_{2}(\mathrm{t})
$$

whilst equation (2) for the production dynamics assumes the scalar form

$$
\dot{\mathrm{x}}(\mathrm{t})=\mathrm{u}_{2}(\mathrm{t})-\mathrm{d}(\mathrm{t}) \quad .
$$

It is assumed that $\tau_{1}=0.5$ and $\tau_{2}=0.5$ so that, in view of the inequalities (3), the production rates must satisfy the constraints $\mathrm{u}_{1}(\mathrm{t}) \leq 2$ and $\mathrm{u}_{2}(\mathrm{t}) \leq 2$. In addition, it foliows from the inequalities (4), (5), and (6) that the state variables and control variables must satisfy the constraints $q_{1}(t), q_{2}(t) \geq 0, u_{1}(t), u_{2}(t) \geq 0$ and $i(t) \geq 0$. The initial state of the system is such that $\mathrm{q}_{1}(0)=5, \mathrm{q}_{2}(0)=0$ and $\mathrm{x}(0)=0$. It is assumed that control is to be exercised on the time interval $[0,10]$, and that the part demand rate on this interval is $d(t)=1 \quad(0 \leq t \leq 10)$. The objective of such control is to choose $i(t), u_{1}(t)$, and $u_{2}(t)$ so as to minimise the cost function in equation (7) with $\mathrm{T}=10, \lambda=[5,10], \mu^{+}=\mu^{-}=5$, and $\mathrm{N}=20$.

The results of solving this problem genetically with a population size $v=200$, a crossover probability $\tau_{c}=0.6$, and a mutation probability $\tau_{m}=0.01$ over 50,000 generations are shown in Figs 1 and 2. In Fig. 1(a) the best-of-generation value of the cost function is plotted against generation number, whilst in Fig. 1(b) the generation-average value of the cost function is plotted. In Figs 2(a), 2(b), and 2(c), respectively, the control variables obtained after 50,000 generations are plotted. These controls give the buffer production rates and the part release rate, and are obtained simply by decoding the best-of-generation design from the last generation of the evolutionary process. It is evident from Fig. 1(a) that several periods of stasis occur (e.g., between $1.1 \times 10^{4}$ and $2.7 \times 10^{4}$ generations, and between $2.7 \times 10^{4}$ and $4.3 \times 10^{4}$ generations) and therefore that the control policies corresponding to such periods are sub-optimal. In fact, even after 50,000 generations, the value of the best-of-generation cost function is 69.39 (compared with the theoretically optimal value of 62.5). However, the sub-optimality is even more pronounced during the period of stasis between $0.7 \times 10^{4}$ and $1.1 \times 10^{4}$ generations. It follows that - if truly optimal performance is required - great care must be exercised in avoiding the sub-optimal control policies corresponding to such periods of stasis. Nevertheless; in this example, the control policies obtained from around 10,000 generations onwards of the evolutionary process are acceptable in practice.

\section{CONCLUSION}

It has been shown that punctuated equilibria can occur in the evolutionary processes associated with the genetic synthesis of optimal control policies for manufacturing systems. The care that must therefore be exercised in avoiding sub-optimal control policies has been illustrated by reference to the genetic synthesis of optimal control policies for a simple manufacturing system.

\section{REFERENCES}

[1]S.B. Gershwin, Manufacturing Systems Engineering, Prentice-Hall, 1994.

[2]J.B. Sousa and F.L. Pereira, "A receding horizon strategy for the hierarchical control of manufacturing systems", Proc. 4th International Conference on Computer Integrated Manufacturing and Automation Technology, 1994, pp 443-450.

[3]B. Porter and C. Allaoui, "Genetic synthesis of optimal control policies for manufacturing systems", Proc. 2nd World Automation Congress, Montpellier, France, May 1996.

[4]B. Porter, "Genetic design of control systems", J.SICE, vol 34, 1995, pp 393-402.

[5]D.E. Goldberg, Genetic Algorithms in Search, Optimization and Machine Learning, Addison-Wesley, 1989.

[6]N. Eldredge, and S.J. Gould, "Punctuated equilibria: an alternative to phyletic gradualism", pp 82-115, Models in Paleobiology (ed. J.M. Schopf), Greeman, Cooper, 1972.

[7]A. Sharifnia, "Stability and performance of distributed production control methods based on continuous-flow models", IEEE Trans. Automatic Control., vol AC-39, 1994, pp 725-737.

[8]K. Egilmez, K. and A. Sharifnia, "Optimal control of a manufacturing system based on a novel continuous-flow model with miniral WIP requirement", Proc. 4th International Conference on Computer Integrated Manufacturing and Automation Technology, 1994, pp 113-118. 


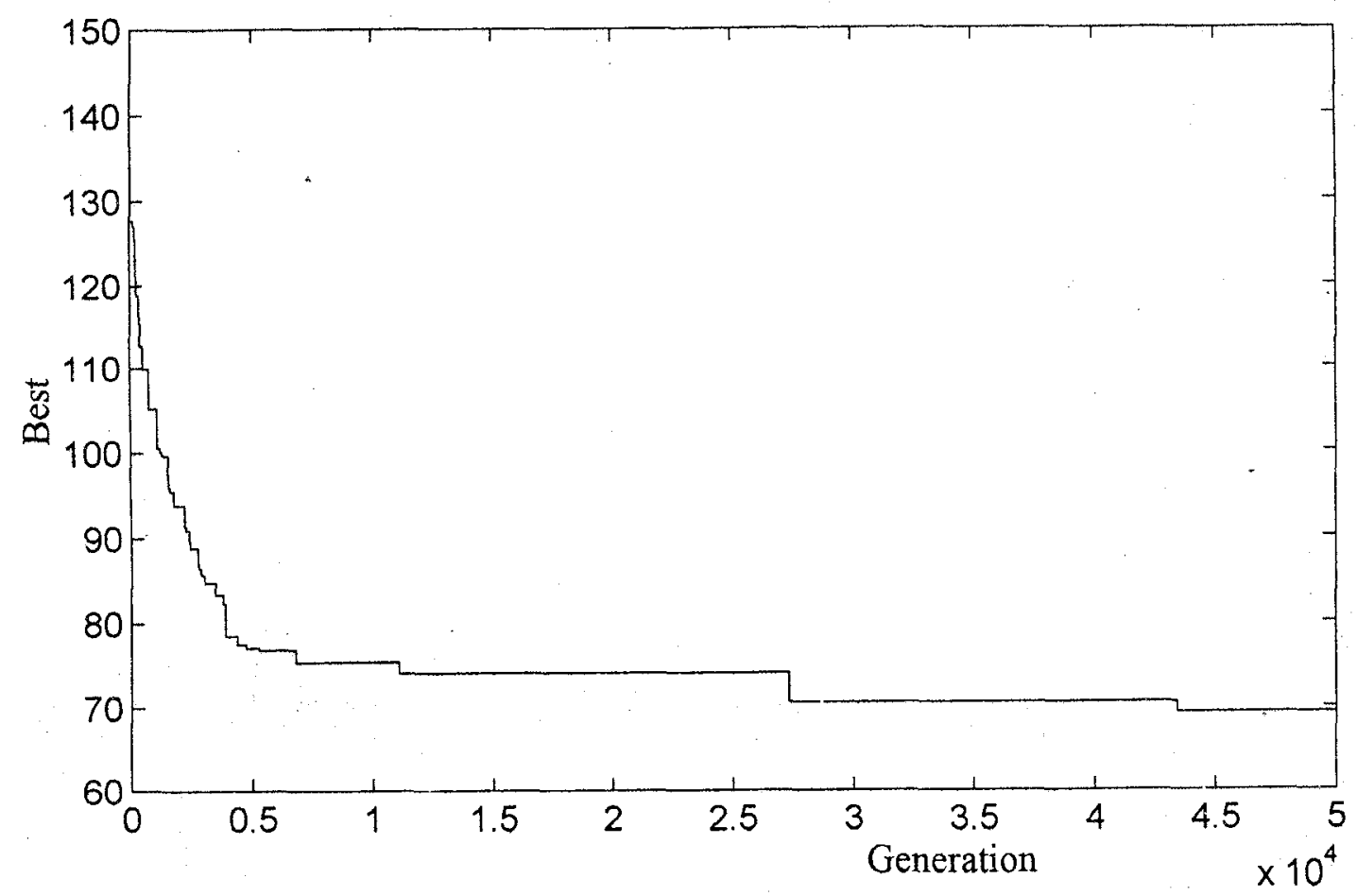

(a)

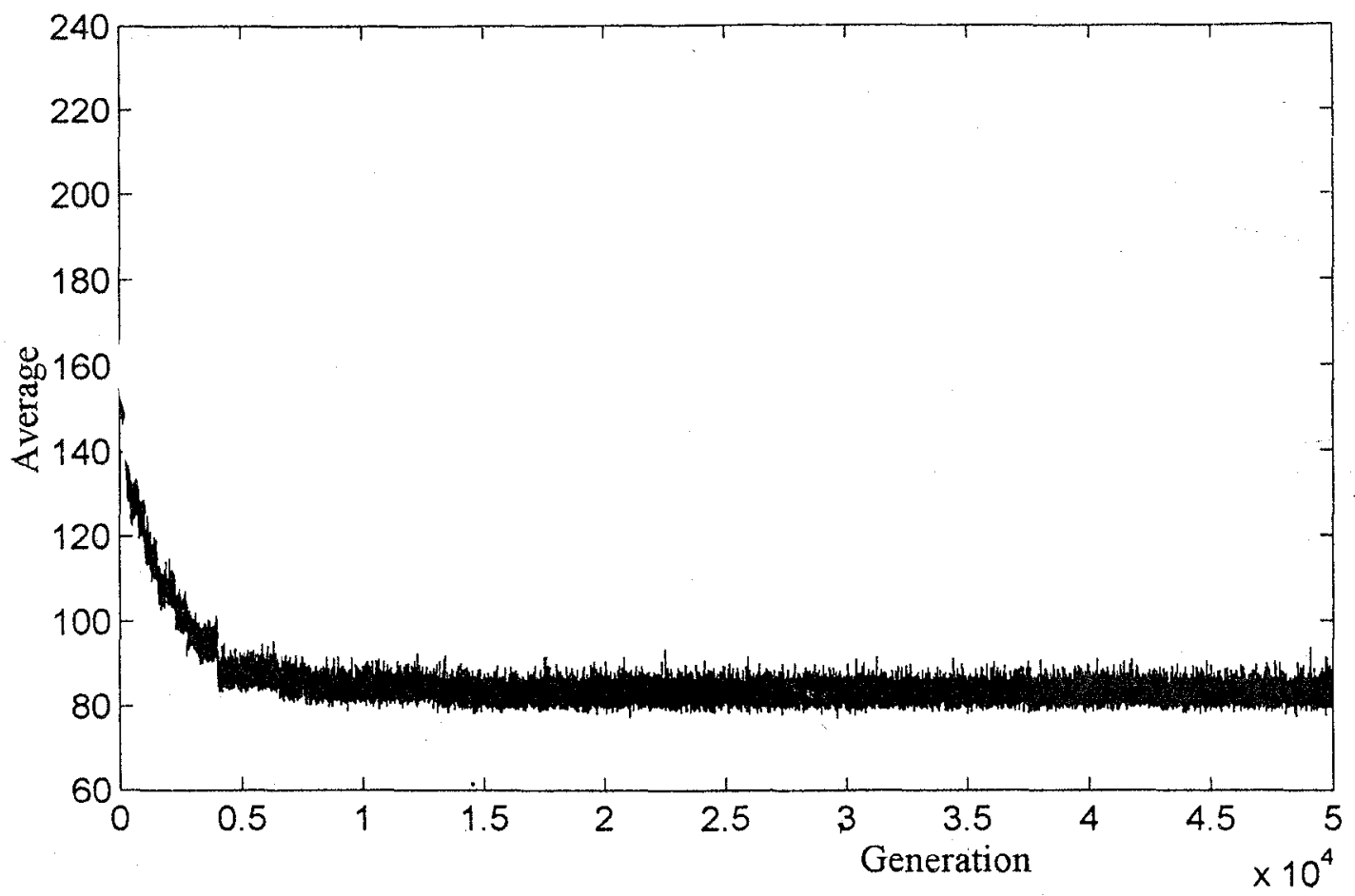

(b)

Figure 1 : Best-of-generation and generation-average of cost function 


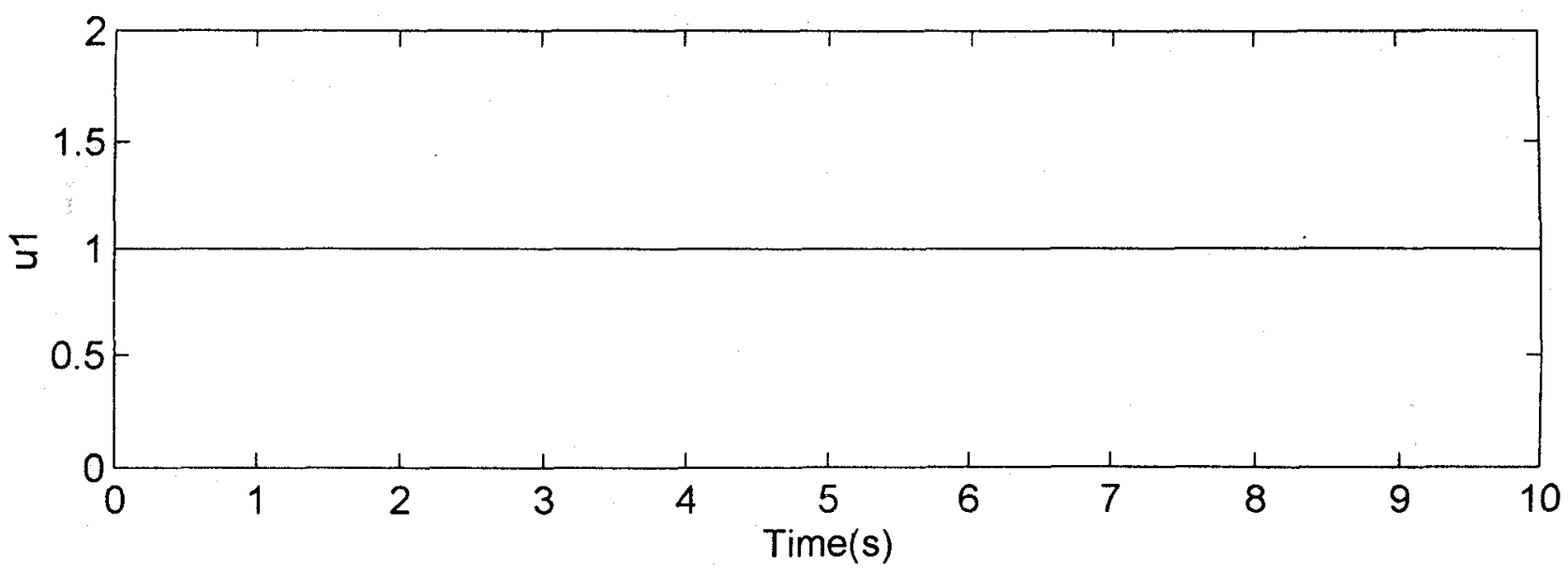

(a)

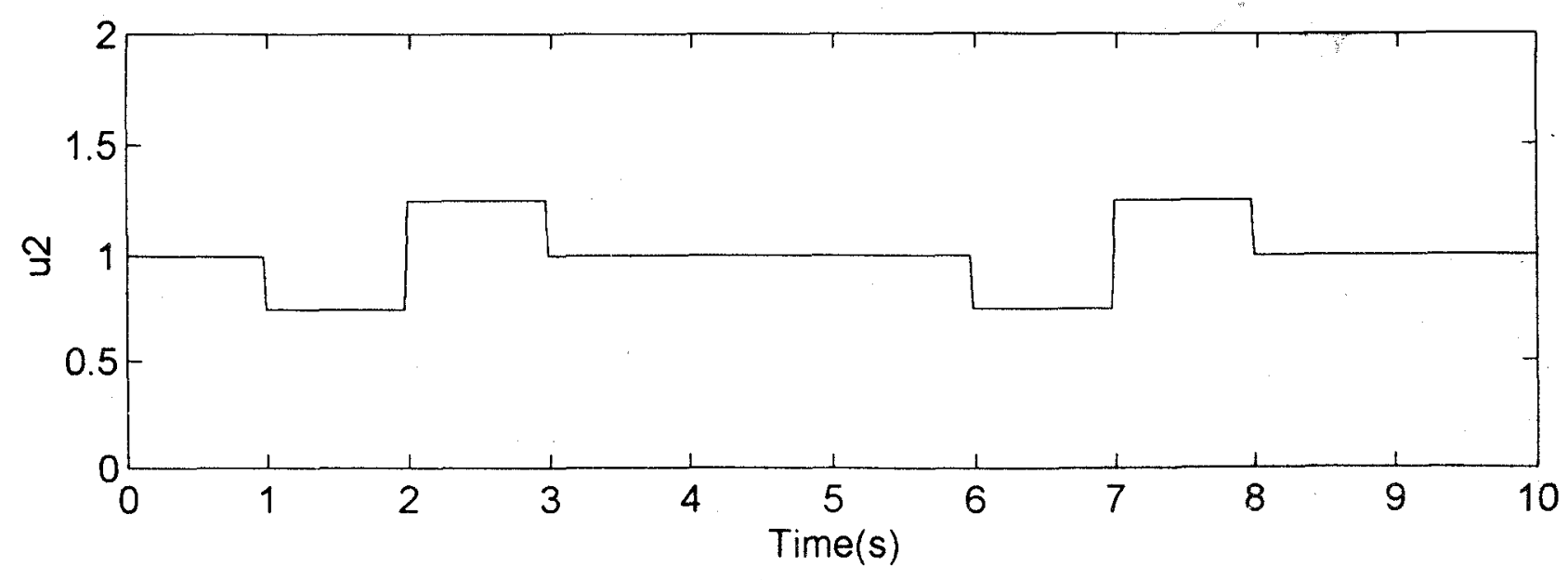

(b)

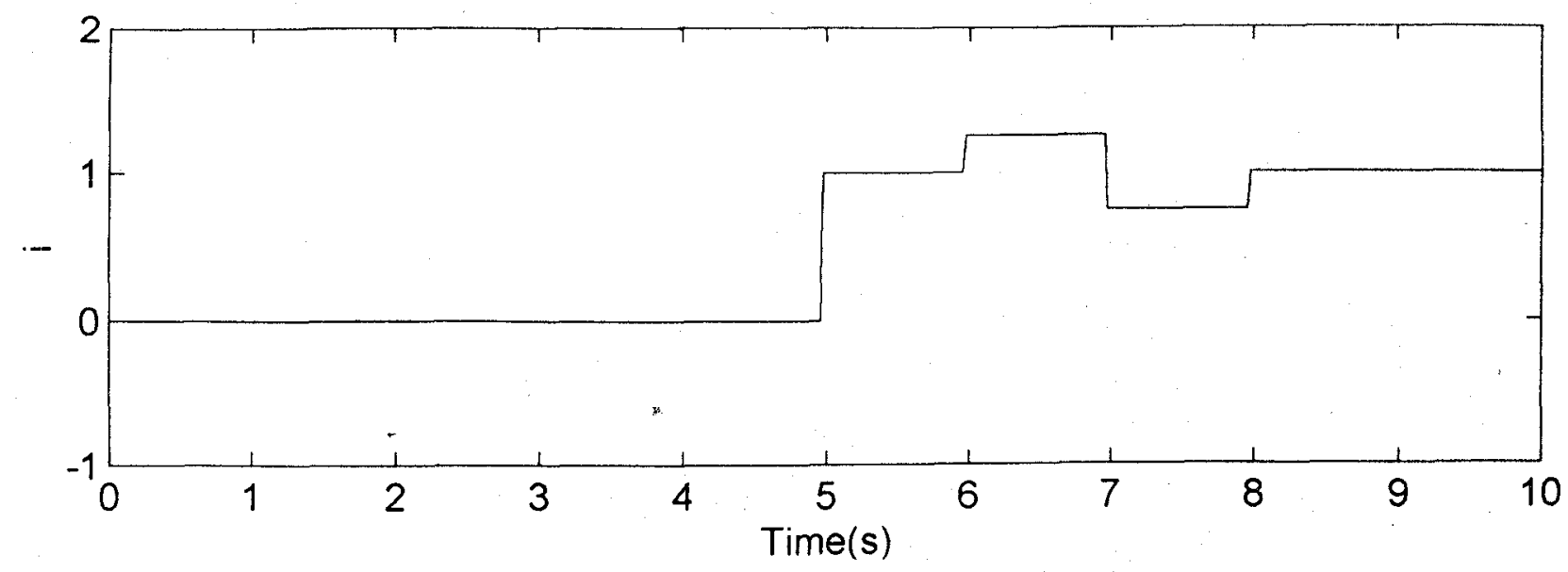

(c)

Figure 2: Control variables after 50000 generations 